Projets

de paysage

\section{Projets de paysage}

Revue scientifique sur la conception et l'aménagement de l'espace

\section{$13 \mid 2015$}

Biodiversité et paysage

\title{
Le bassin minier du Nord-Pas-de-Calais. Un paysage stratifié entre projets, amnésies et créations
}

Retour sur une démarche de recherche interdisciplinaire

The Mining Region of the Nord-Pas-de-Calais. A Composite Landscape of

Projects, Forgotten Heritages, and Designs - Analysing an Interdisciplinary

Research Approach

Fabrice Raffin, Antoine Luginbühl et Olivier Pollet

\section{OpenEdition}

\section{Journals}

Édition électronique

URL : http://journals.openedition.org/paysage/10018

DOI : $10.4000 /$ paysage. 10018

ISSN : 1969-6124

Éditeur :

École nationale supérieure du paysage de Versailles-Marseille, Institut national des sciences appliquées Centre Val de Loire - École de la nature et du paysage, École nationale supérieure d'architecture et de paysage de Bordeaux, École nationale supérieure d'architecture et de paysage de Lille, Agrocampus Angers

\section{Référence électronique}

Fabrice Raffin, Antoine Luginbühl et Olivier Pollet, « Le bassin minier du Nord-Pas-de-Calais. Un paysage stratifié entre projets, amnésies et créations », Projets de paysage [En ligne], 13 | 2015, mis en ligne le 31 décembre 2015, consulté le 04 septembre 2020. URL : http://journals.openedition.org/ paysage/10018; DOI : https://doi.org/10.4000/paysage.10018

Ce document a été généré automatiquement le 4 septembre 2020

Projets de paysage 


\section{Le bassin minier du Nord-Pas-de- Calais. Un paysage stratifié entre projets, amnésies et créations}

Retour sur une démarche de recherche interdisciplinaire

The Mining Region of the Nord-Pas-de-Calais. A Composite Landscape of

Projects, Forgotten Heritages, and Designs - Analysing an Interdisciplinary

Research Approach

Fabrice Raffin, Antoine Luginbühl et Olivier Pollet

1 Située au cœur du bassin minier Nord-Pas-de-Calais sur la partie sud de l'aire métropolitaine de Lille, la communauté d'agglomération d'Henin-Carvin ${ }^{1}$ (CAHC) partage son histoire avec celle de l'exploitation minière. Inscrit au patrimoine mondial de l'Unesco, ce territoire constitue pourtant une entité paysagère aux multiples facettes dont il s'agit ici de saisir les interactions et la complexité dès lors que l'on considère le paysage comme un projet ${ }^{2}$, un analyseur et un processus qui lie tout à la fois la nature et l'humain, le politique et l'économique, la culture et l'esthétique, autant que l'écologique et l'aménagement ${ }^{3}$.

2 Après avoir présenté les enjeux méthodologiques d'une analyse de la dimension paysagère des projets d'aménagement du territoire, le texte détaille cinq dynamiques paysagères de cette partie $d u$ bassin minier, entre passé minier, enjeux environnementaux et développement récent de l'activité économique de logistique des marchandises sur le site de la plateforme multimodale Delta 3 à Dourges, notamment.

Ce texte se fonde sur la recherche que nous menons depuis trois ans, dans le cadre du programme Infrastructures de transports terrestres, écosystèmes et paysage (Ittecop) ${ }^{4}$ (Medde-Ademe). 


\section{Le projet de paysage : acteurs, échelles, temporalités}

Une des caractéristiques de la recherche dont nous rendons compte ici est d'avoir rassemblé une équipe de paysagistes et une équipe de sociologues. À une définition commune du paysage comme projet dont les paysagistes avaient la méthodologie permettant de le caractériser, les sociologues ajoutaient une interrogation sur les processus de mise en œuvre de ce projet. Plus précisément, il s'agissait de caractériser ce projet par l'identification de ses acteurs, ses échelles de déploiement et ses origines temporelles plus ou moins lointaines. Rencontre dont l'enjeu méthodologique n'était pas sans rappeler les différentes approches du paysage qu'évoquait Isaac Joseph dès les années 1990 entre l'ordre factuel et matériel du paysage et l'ordre sensible et symbolique (Joseph, 1998)5. L'enjeu de la rencontre entre paysagistes et sociologues était bien de « sortir de l'opposition grossière de l'objectif et du subjectif » (ibid.).

De ce point de vue, la diversité des compétences rendait possible la prise en compte d'une diversité d'échelles d'observations et de questionnements. Il s'agissait de prendre en compte la complexité du territoire observé pour parvenir à une vision d'ensemble permettant de mettre en lien toutes les activités qui l'ont modelé, le modèlent et le modèleront. En particulier, une analyse paysagère et socioanthropologique de la communauté d'agglomération et des espaces logistiques permettait une reconstruction et une analyse du processus d'émergence, de structuration et de construction de ces espaces et de leurs paysages ${ }^{6}$.

\section{Cadre méthodologique}

"Lorsque nous abordons un groupe, un collectif, une communauté, ce sont les interactions et les interdépendances qui les lient aux autres qui font au mieux sens pour nous : il est des démarches de recherche, à tel point respectueuses de la singularité culturelle des formations sociales, qu'elles les enferment sur ellesmêmes, rabattent leurs lisières dans le centre, effaçant ainsi toutes les porosités, les adhérences des uns aux autres » (Tarrius, 1997).

La démarche qualitative de cette recherche s'inscrit dans la tradition d'une socioanthropologique urbaine (Hannerz, 1983 ; Davis, 2006 ; Tarrius, 1992). Dans notre approche, nous ne donnons pas d'exclusivité a priori aux acteurs légitimes de l'aménagement urbain, mais nous procédons à une mise à plat de la pluralité des acteurs et de leurs logiques, ainsi que des interactions qu'ils entretiennent entre concurrences, conflits, tensions, coopérations, accommodations, voire indifférence, ignorance, évitement. Les données proviennent de 10 campagnes d'investigation de terrain réalisées entre 2012 et 2015, visant à couvrir le territoire des 14 communes de la CAHC. Chaque campagne de terrain mobilisait entre deux et quatre chercheurs à plein-temps durant deux à cinq jours. Ces campagnes de terrain ne se limitaient pas à la passation d'entretiens. Leur objectif principal visait à aller au plus près des réalités quotidiennes ou plus exceptionnelles du territoire : arpenter « non-stop » le territoire, d'un entretien à l'autre, d'un site ou d'une visite à l'autre, d'un imprévu à un intérêt soudain. Au-delà de la liste de la cinquantaine d'entretiens formels qui ont fait l'objet d'un travail d'analyse approfondie, cet arpentage consistait également à de multiples rencontres, discussions en des lieux aussi nombreux qu'imprévus. D'autre part, suivant les 
réseaux d'acteurs et leurs logiques, plusieurs campagnes de terrain " délocalisées » et complémentaires ont été réalisées afin d'enrichir nos questionnements et d'appréhender les échelles de déploiement des processus observés. Si les notions de représentativité et d'exhaustivité ne sont pas au cœur de notre démarche socioanthropologique, nous fondons le caractère scientifique de notre analyse sur le « principe de saturation » propre aux sciences sociales (Pétonnet, 1979 et 1982 ; Delaporte, 1993). C'est-à-dire une observation des faits jusqu'à stabilisation de leur redondance, doublée de la répétition systématique des faits observés (pratiques, usages de l'espace notamment) et des discours enregistrés. Le point de saturation est atteint lorsque le chercheur s'est efforcé de diversifier le plus possible ses informateurs et que l'impression de ne plus rien apprendre de nouveau s'installe, du moins en ce qui concerne l'objet sociologique de l'enquête (Bertaux, 1997 ; Demaziere et Dubar,1997 ; Beaud,1996.

\section{Questionner le projet de paysage. Une pluralité enchevêtrée}

Dès lors, l'hypothèse qui se dégageait de nos travaux était que le processus continu de production des paysages relève moins de la mise en œuvre d'un projet que de la succession, de l'entremêlement de projets de territoire portés par différents acteurs en interaction. Des acteurs qui ne sont pas toujours politiques, mais qui sont principalement focalisés sur des enjeux de développement économique. Des acteurs qui, surtout ici, traitent de manière secondaire les enjeux paysagers, inscrits dans différentes profondeurs historiques et articulant différentes échelles.

L'évolution des processus paysagers pour la CAHC renvoie ainsi à différentes strates temporelles dominées chacune par un modèle économique, du Moyen Âge à nos jours. $\mathrm{Au}$ cœur de ce processus, les évolutions récentes font apparaître une dimension de projet de paysage définie par le volontarisme et des choix économiques des élites politiques locales. Comme nous allons le montrer, ce volontarisme de projet évolue néanmoins en interaction avec les héritages passés et d'autres acteurs locaux pour redéfinir le paysage à venir. Projet avant tout économique, il dialogue avec l'économie passée, liant tout à la fois l'aménagement du territoire, les questions écologiques, les transports, les modifications agricoles, le tourisme, comme la vie quotidienne. À travers les aménagements paysagers, c'est ainsi la nature des relations de l'homme à son environnement qui est convoquée, mais également la nature des rapports sociaux entre différents groupes, à différentes échelles.

\section{Questionner les acteurs des projets de paysage. Une multiplicité en interaction}

8 Concernant ces acteurs des projets de paysage, il convient de poser un préalable à notre approche qui rompt avec beaucoup de perceptions contemporaines communes de l'aménagement du territoire. Ce que montre notre recherche et beaucoup de travaux récents est que les acteurs des projets de paysage ne se limitent pas à un seul groupe social, serait-il celui des élus7 ${ }^{7}$. Comme le rappelle Marie-Flore Mattei, « ce qui "fait" la ville résulte, certes, de volonté politique, d'opérations d'urbanisme (ZAC, lotissements, grands projets...), mais surtout de décisions privées ${ }^{8}$ ». De fait, comme c'est le cas sur la CAHC, les opérations d'urbanisme et autres grands projets mobilisent toujours des 
acteurs publics (élus, mais également techniciens, acteurs du patrimoine notamment et beaucoup d'autres) et des acteurs privés de différents statuts : acteurs économiques (promoteurs, acteurs de la grande distribution, de la logistique, agriculteurs, petits commerçants et artisans, pour les principaux sur la CAHC), acteurs associatifs, riverains, etc. Pour le dire autrement, les acteurs du paysage sont toutes les personnes susceptibles de se constituer comme " public $^{9}$ » d'une question d'aménagement au sens de Daniel Céfaï (2013). Il n'y a donc pas deux catégories d'acteurs, mais une multitude en nombre variable, en fonction des situations problématiques ou non de l'aménagement, d'enjeux paysagers bien sûr, mais aussi d'enjeux économiques, politiques, patrimoniaux, de voisinages, culturels, etc. Ainsi, le processus paysager est avant tout le résultat des interactions entre ces acteurs, qui en négocient les contenus, les formes, les limites et une part des usages. De ce point de vue, le processus paysager ou les projets se définissent avant tout comme des processus de négociation dont les contenus ne cessent d'évoluer jusqu'à une cristallisation: les réalisations paysagères qui marqueront le début des usages.

Figure 1. Différentes composantes structurantes du paysage de la CAHC héritées et entremêlées

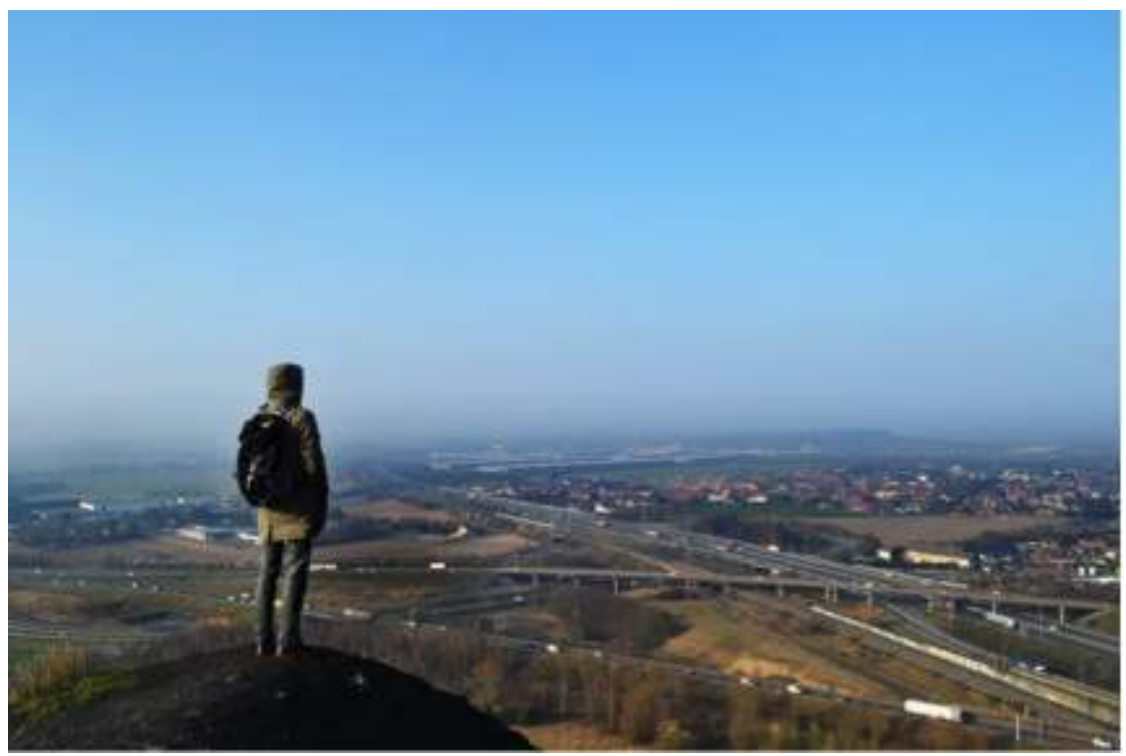

L'agriculture, l'activité minière, l'activité logistique (voies de transport et stockages des marchandises), aménagements environnementaux à vocation paysagère, métropolisation.

Source : Passeurs - Jérémy Pierra.

Le projet de paysage se présente ainsi comme une action collective à différentes dimensions, mobilisant plusieurs types et réseaux d'acteurs, aux ramifications en constante évolution. Les projets de paysage ne résultent pas d'une simple décision politique et aucun groupe social en particulier n'en a la maîtrise complète sur un territoire. Comme le souligne en effet Isaac Joseph (1998) : « Modification du paysage et modifications d'usages vont de pair et traduisent l'ouverture du territoire local, qu'il soit central ou périphérique, à l'ordre des réseaux. » 


\section{Questionner les échelles spatiales des projets de paysage : la notion de dispositif paysager}

Les premières impressions ressenties en parcourant le territoire de la CAHC peuvent être confuses, brouillées par le manque remarquable de repères visuels paysagers clairement identifiés. La CAHC se présente comme une "conurbation» quasiment ininterrompue sur plus de vingt kilomètres, sorte de longue sortie de ville, de Lille en l'occurrence, aux espaces diffus dont la grammaire appréhendée le plus souvent par l'autoroute est peu lisible. L'entrelacement d'infrastructures linéaires, autoroutes, rails, canaux y est frappant. Pour peu que l'on s'y arrête cependant, les caractères paysagers apparaissent plus clairement, indiquant différentes strates historiques et dynamiques à l'œuvre.

11 En effet, ayant traversé plusieurs périodes de développement économique monospécifique, le territoire a supporté différentes dynamiques entraînant l'évolution de ses paysages de façon très marquée. Entre économies agricole et minière ou aujourd'hui économie de la logistique, un enchevêtrement de modèles économiques successifs a marqué les représentations sociales du territoire. Mais cette représentation est en évolution sur la base des dynamiques paysagères en cours, qui s'illustrent à travers des logiques de projet dont les acteurs du territoire et les paysages sont à l'origine, de l'acteur du local à l'acteur du global.

12 Ainsi, à travers les réseaux du processus et les réalisations paysagères, les étapes qui construisent le projet de paysage mobilisent différentes échelles pour le constituer. Il est habituel d'interroger les échelles spatiales des projets de paysage, les échelles de leur insertion urbaine en l'occurrence, dont les limites ne sont pas toujours précises. Mais la question des limites est ici appréhendée par la prise en compte des usages réels des aménagements après leur construction. Emboîtement des paysages à travers les déplacements et les usages de l'espace, construction du paysage dans la mobilité ${ }^{10}$ et emboîtement des cadres et des unités paysagères, telle serait l'approche socioanthropologique du paysage qui définirait des entités successives.

13 Cette manière de rendre compte de paysages successifs ne facilite pas la tâche du paysagiste plus habitué à présenter des unités paysagères relativement homogènes ou à repérer les homogénéités et cohérences d'un paysage. La solution pour analyser pleinement cette diversité renvoie à la notion de dispositif paysager, entendu comme une articulation d'entités paysagères dont le lien est moins réalisé par leurs caractéristiques matérielles objectives et sensibles que par les pratiques et usages de l'espace des habitants. Le paysage contemporain de la CAHC se présente ainsi comme l'articulation de paysages de la logistique des marchandises (plateformeDelta 3, entrepôts Amazon, ainsi qu'un ensemble de plus petites entreprises privées), des paysages du commerce de masse (ZAC du Bord-des-Eaux-centre commercial AuchanNoyelles à Noyelles-Godault, centre commercial Maison Plus-Ikea à Henin Beaumont), des paysages eux-mêmes en lien avec les héritages passés, agriculture, mines, villecentre, etc. 


\section{Questionner les échelles temporelles du paysage : entre passé, présent et avenir}

De ce dernier point de vue historique, au-delà de la question des échelles spatiales, la question des échelles temporelles du paysage est plus rarement prise en compte. Pourtant, si l'on peut parler de stratification paysagère, c'est bien à partir de l'accumulation des héritages paysagers passés, ces derniers renvoyant aux activités économiques principalement. Lorsque ces activités cessent, comme l'activité minière en Nord-Pas-de-Calais, elles ne s'effacent pas du paysage, elles en sont toujours constitutives. De plus, au-delà d'éléments paysagers, ce sont également les rapports sociaux passés qui structurent les projets de paysages présents et à venir.

Ainsi, par la prise en compte entrecroisée des espaces et du temps spécifiques de la CAHC, notre analyse fait apparaître cinq grandes dynamiques paysagères synthétisées dans la figure ci-dessous.

Figure 2. Les grandes dynamiques en cours constituant les paysages actuels du territoire de la CAHC

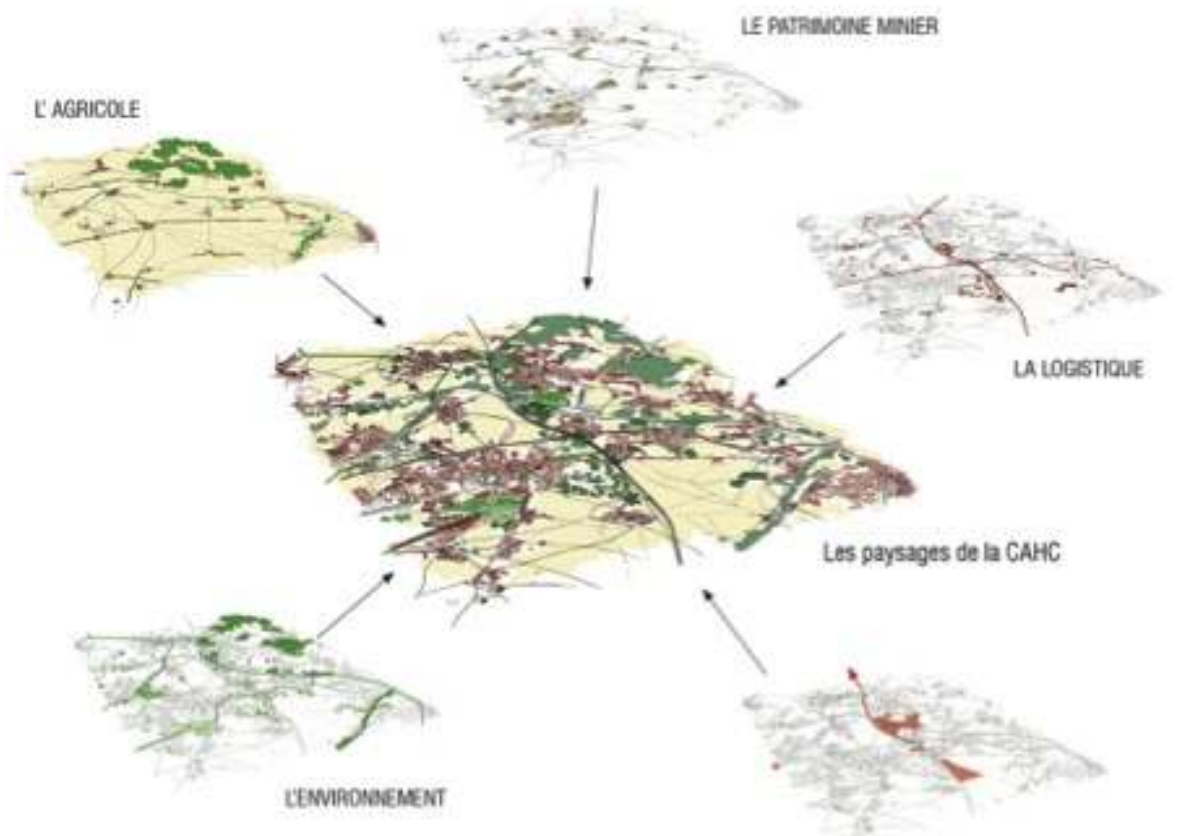

LAMETAOPOUSATION

Source : Passeurs - Antoine Luginbühl.

\section{La combinaison des cinq dynamiques des paysages de la CAHC}

D'un modèle agricole jusqu'à la moitié du XIX ${ }^{e}$ siècle, le territoire est passé à un modèle industriel essentiellement marqué par l'exploitation minière, dont l'activité a perduré jusqu'à la fin du $x^{e}$ siècle. Aujourd'hui, l'activité industrielle étant déclinante, le territoire semble avoir trouvé un nouveau modèle de développement économique basé 
sur la logistique. Mais ces modèles économiques monospécifiques n'ont pas pour autant totalement effacé les modèles précédents. L'agriculture a toujours subsisté en tant que ressource économique, bien que beaucoup moins génératrice d'emplois. L'exploitation minière, bien que totalement stoppée, produit encore quelques emplois liés au domaine culturel et à la valorisation de son passé encore très présent dans la mémoire collective.

17 Aujourd'hui, ces périodes de l'histoire du bassin minier ont laissé des traces et des éléments encore en activité dont les paysages actuels sont composés. Le palimpseste du paysage s'exprime très clairement à travers des strates plus ou moins perceptibles. Chaque modèle de développement, que l'on peut associer à des périodes de l'histoire, a généré des dynamiques paysagères qui ont façonné les paysages actuels. À ces dynamiques héritées, il faut également ajouter un modèle contemporain environnemental ou écologique (préservation des écosystèmes, de la biodiversité, etc.), ainsi que celui lié au phénomène de métropolisation. Cet entremêlement de modèles économiques successifs et de représentations sociales du bassin minier est encore à l'œuvre de manière plus ou moins affirmée selon les différentes dynamiques paysagères en cours. Ces dernières s'illustrent à travers des logiques de projet dont les acteurs du territoire sont à l'origine.

\section{Un territoire agricole jusqu'à la fin du XIX ${ }^{\mathrm{e}}$ siècle}

18 Jusqu'au XIX ${ }^{e}$ siècle, le territoire était largement façonné par l'agriculture. Les paysages actuels sont constitués d'éléments hérités de ce modèle agricole, entre un urbanisme spécifique et les terres cultivées. Aujourd'hui, les paysages agricoles semblent ne représenter qu'une toile de fond du territoire de la CAHC aux yeux de la plupart des acteurs territoriaux, bien que l'agriculture reste très dynamique. La présence de ces paysages agricoles aux lisières de la grande conurbation Béthune-Valenciennes confère au territoire de la CAHC un caractère fortement "périurbain ». En effet, comme une grande partie du territoire national, le bassin minier a connu une forte extension de la surface urbanisée depuis la seconde moitié du XIX siècle.

Figure 3. Terres agricoles sur la commune de Courcelles-lès-Lens

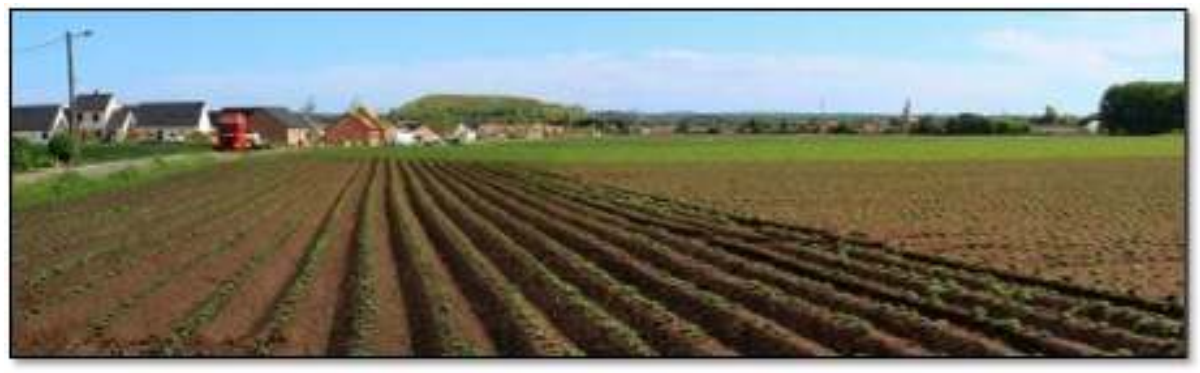

Le développement du bassin minier n'a pas effacé la trame agricole et rurale.

19 La grande majorité de ces extensions urbaines périphériques est caractérisée par un tissu résidentiel de constructions individuelles basses sous forme de lotissement. Cette urbanisation se fait aux dépens de la surface agricole utile, en proie à toutes les problématiques connues de déplacement de ménages urbains vers les espaces ruraux. Sur le territoire de la CAHC, l'urbanisation profite en majorité aux populations issues du bassin minier et, en plus petite proportion, aux citadins de la métropole lilloise. 
S'opposant à ce phénomène d'artificialisation des terres, un certain nombre d'agriculteurs se mobilisent pour proposer une alternative au mode de consommation alimentaire. De nombreuses exploitations ouvrent leurs portes pour vendre les produits issus directement de la ferme. Contrairement au phénomène de consommation alternative connu dans les grandes villes, ce sont les populations locales issues des catégories sociales populaires qui profitent de cette nouvelle offre.

D'autre part, les bâtiments agricoles de la CAHC présentent la particularité d'être regroupés autour des rues des bourgs, formant des «villages-rues ». Les traces de ces exploitations agricoles, lorsqu'elles ne sont plus en activité, sont très présentes dans le paysage. On les reconnaît à leur plan et à leur architecture, notamment à la porte cochère donnant sur la rue et faisant office d'entrée principale de l'exploitation. La seconde particularité est leur positionnement entre le village et les terres cultivées. Les traversées permettent de passer du cœur du village aux champs cultivés, de manière générale positionnés à l'arrière et attenants à l'exploitation. Le parcellaire en lanière illustre bien cette caractéristique.

Figure 4. Un urbanisme lié aux pratiques agricoles encore très présent sur le territoire de la CAHC

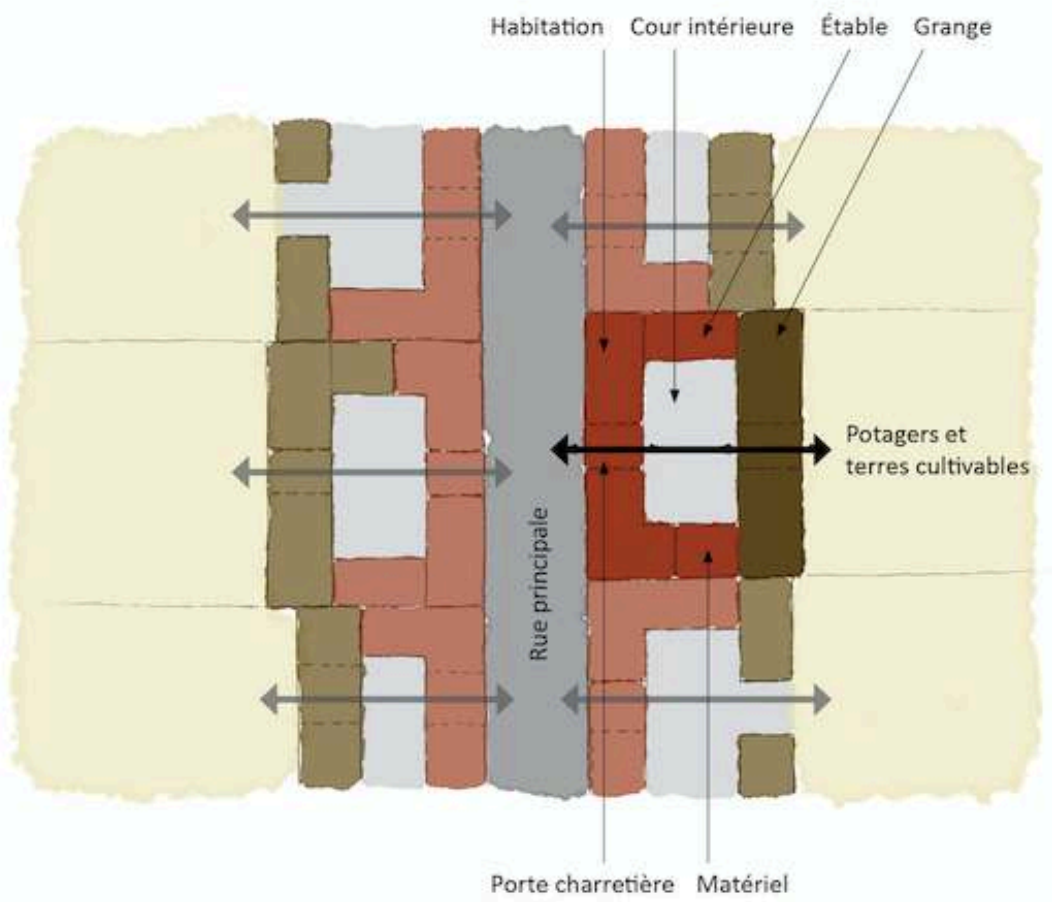

Source : Antoine Luginbühl.

\section{L'exploitation minière, un bouleversement majeur des paysages du territoire}

21 À partir de la seconde moitié du XIX ${ }^{e}$ siècle, les paysages du Pas-de-Calais connaissent un bouleversement majeur par l'implantation des exploitations minières, des cités ouvrières et d'un réseau de «cavaliers ${ }^{11}$ ». Les terrils s'élèvent peu à peu jusqu'à la fin $\mathrm{du} \mathrm{xx}^{\mathrm{e}}$ siècle pour constituer ce que l'on appelle aujourd'hui de manière évidente le 
"bassin minier». Son fonctionnement est lié à son contexte géographique et géologique particulier, ayant conduit à deux siècles et demi d'histoire de l'extraction minière, histoire dont les paysages de la CAHC gardent encore de nombreux stigmates. Dès le $\mathrm{XIX}^{\mathrm{e}}$ siècle, la logique d'aménagement «fosse-terril-cité » s'est imposée comme unique schéma de développement en rupture avec l'organisation agricole traditionnelle prédominante jusqu'alors. Les bourgs, historiquement ruraux et composés de fermes d'exploitation, se voient modifiés et de nouvelles centralités urbaines sont créées.

Figure 5. Jouxtant les communes d'Hénin-Beaumont et Noyelles-Godault, situé au bord de l'autoroute A1 et de la voie du TGV Nord, le terril Sainte-Henriette est particulièrement exposé

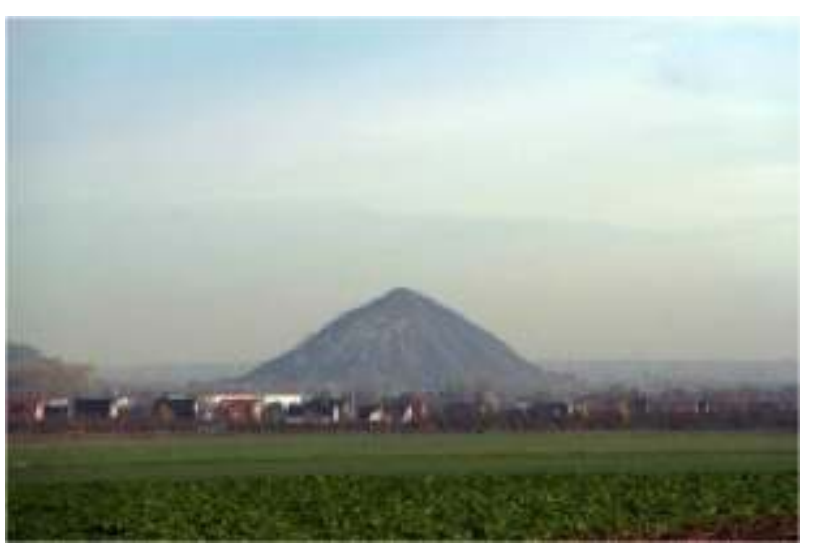

Outre un impact paysager important, les terrils ont un rôle de refuge pour la faune et la flore. Source : Antoine Luginbühl.

Depuis une quinzaine d'années, une mise en valeur progressive des éléments liés à l'exploitation minière est à l'œuvre. Cette combinaison de dynamiques, basée sur une reconquête environnementale, mais aussi patrimoniale et culturelle de ces éléments, tente d'améliorer la qualité de vie sur l'ensemble du territoire et participe du changement de son image. La représentation de l'héritage minier a évolué en une quinzaine d'années. Elle est passée d'une vision de «séquelle " à celle d'un héritage, puis à celle d'un patrimoine culturel. Engagé dès le début des années 2000, ce processus de patrimonialisation va se trouver renforcé le 30 juin 2012 avec l'inscription du bassin minier au patrimoine mondial de l'humanité par l'Unesco au titre de « Paysage culturel évolutif vivant ». Trois enjeux sous-tendent cette politique de mise en patrimoine: celui de la reconnaissance de l'héritage minier (patrimoine matériel et immatériel) comme fondement culturel du territoire, celui de contribuer au changement d'image du territoire qui pâtit encore, vingt ans après la fermeture du dernier puits de mine, d'une image dévalorisée, et enfin celui de favoriser la place de la culture et du patrimoine minier et industriel au sein de dynamiques de développement local et régional.

Au cœur du processus de revalorisation, pour nos interlocuteurs, il est notable que la reconversion des éléments miniers doive obligatoirement s'acquitter d'une dimension écologique. Ce facteur freine d'ailleurs beaucoup leurs prises de décision d'aménagement du territoire. Car si les projecteurs sont braqués sur un ou deux éléments patrimoniaux emblématiques, une des difficultés de la mise en place de la politique de reconversion tient à la représentation négative de la population à l'égard de l'héritage minier. Les terrils sont en effet soumis à une double lecture sociale : alors que les habitants lillois se représentent généralement les terrils comme des "verrues » 
paysagères, les habitants du bassin minier les considèrent dans leur majorité comme des "outils de travail", reliquats de la période révolue de l'exploitation minière. L'habitat minier fait également l'objet de perceptions et de visions contrastées. Reconnues pour leur confort avant-gardiste et leurs qualités architecturales lors de leur construction, et labellisées depuis patrimoine mondial de l'Unesco, les cités minières sont perçues comme exiguës et peu confortables. Le discours notamment des populations jeunes du bassin minier est particulièrement révélateur de représentations négatives des cités minières parfois assimilées à des ghettos ou à une certaine soumission et liées à une mémoire qu'ils disent ne pas être la leur.

\section{Des politiques successives en vue d'une reconquête environnementale du bassin minier, le « passage du gris au vert »}

Dès le XIX ${ }^{e}$ siècle, les croissances conjuguées des différentes activités industrielles liées à la mine (sidérurgiques notamment) se sont révélées très préjudiciables à l'environnement. La spécificité et l'intensité des problèmes du bassin minier en font un territoire d'attention prioritaire et de requalification des friches industrielles. Des années 1970 à aujourd'hui, nombreux ont été les programmes lancés pour inverser la tendance. La requalification du réseau de cavaliers constitue un axe important du schéma de reconversion des anciens espaces dévolus à l'activité minière. S'appuyant sur ce réseau comme alternative de déplacement, mais aussi comme support d'activités récréatives (VTT, promenade, randonnée...), ce sont plus de 120 kilomètres d'anciennes voies ferrées en friche qui ont été reconvertis progressivement. Aujourd'hui, le projet de la chaîne des parcs ambitionne de relier les espaces industriels et les trames vertes et bleues, en s'appuyant sur ce réseau. La transformation d'une ancienne cokerie en grand parc "naturel» urbain, le parc des îles de Drocourt, illustre cet objectif de reconquête environnementale. La protection du patrimoine et l'inscription des terrils dans la politique des espaces naturels sensibles (ENS) du conseil général du Pas-deCalais participent du mouvement de réhabilitation du bassin minier et de revalorisation de son image. L'ancien « pays noir » devient « vert » et se transforme progressivement en un "cadre privilégié pour les randonneurs et amoureux de grands espaces", selon les mots des acteurs territoriaux. Au total, en l'espace de 30 ans, plus de 8000 hectares de friches industrielles ont été reconquis.

Dans la perspective d'un changement d'image de la CAHC, plusieurs initiatives illustrent la volonté de valorisation de l'ensemble du patrimoine vert existant. Cette nouvelle tendance se manifeste dans la protection des espaces naturels sensibles et par la mise en valeur des trames vertes et bleues. La dépollution des sites industriels est un axe fort de la politique de reconversion du bassin minier partagé par tous, politiques, techniciens et populations locales. 
Figure 6. Évolution des logos, de la charte graphique et des objets de communication de la CAHC

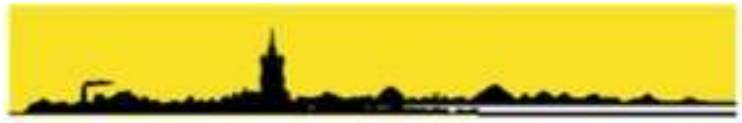

COMMUNAUTÉ D'AGGLOMÉRATION D'HÉNIN-CARVIN

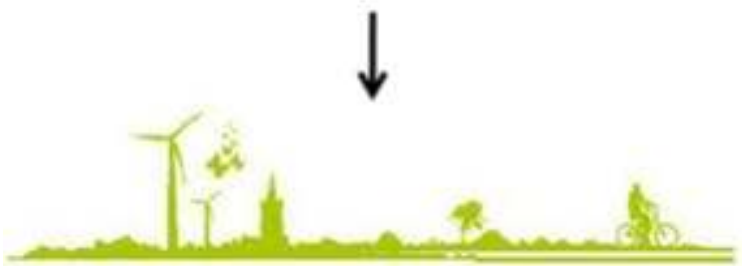

\section{Communautẻ d'Agglomération Hénin-Carvin}

Cette évolution des logos (représentés ici avant et après les années 2000-2005 d'après nos recherches), de la charte graphique ainsi que des objets de communication de la CAHC illustre la volonté de renouvellement d'une image d'un territoire industriel, pollué et socialement dégradé, à celle d'un territoire « vert » soucieux de son environnement et de son équilibre social.

Source : CAHC (logos issus du site web www.agglo-henincarvin.fr)

Au-delà d'une question d'image, il s'agit d'une nécessité incontournable pour envisager un tournant dans le développement du territoire. Nécessité physique pour retrouver une activité au sein des sites industriels, mais aussi morale pour oublier un passé difficile et inciter les jeunes générations à rester sur le bassin ${ }^{12}$. On peut donc parler d'une "dépollution physique et morale ». L'exemple le plus symbolique est sans doute celui du site de Metaleurop, dont la «renaturation» a fait l'objet d'un lourd investissement financier.

\section{La dynamique de métropolisation et le développement des paysages de la logistique}

Le positionnement de la CAHC au sein d'un espace métropolitain dynamique considéré comme le "carrefour de l'Europe ", densément peuplé et urbanisé, traversé par de nombreux axes de circulation majeurs, lui confère des caractéristiques et problématiques paysagères singulières. Le développement d'un grand parc naturel urbain sur le site de l'ancienne cokerie de Drocourt, la création d'un nouveau quartier mixte sur le site de Sainte-Henriette, le projet de liaison RER entre la métropole lilloise et le bassin minier, la reconversion de la fosse du 9/9 bis en lieu de créativité et d'activités (musique, tourisme d'affaires, excellence logistique), sont autant de projets qui témoignent de l'inscription de la CAHC dans une dynamique de développement qui dépasse ses limites territoriales pour se placer à l'échelle de l'aire métropolitaine de Lille.

Occupant une position favorable au cœur des flux de transport européen, le Nord-Pasde-Calais s'affirme progressivement comme un hub «marchandises» de l'Europe du Nord-Ouest. L'implantation en 2003 d'une infrastructure de transbordement de marchandises à vocation européenne, la plateforme multimodale Delta 3 à Dourges sur le territoire de l'ancien bassin minier, traduit et conforte l'ambition partagée des acteurs du territoire d'en faire un nouvel « eldorado » de la logistique.

Cette dynamique se met à l'œuvre dans les années 1990, à la suite de l'arrêt de l'activité minière. Entre la nécessité de remplacer un modèle de développement en crise et la 
volonté de tourner la page d'une période "noire" révolue, le modèle de développement économique basé sur la logistique apparaît comme porteur d'avenir. Perçue comme «non polluante », «créatrice d'emploi » et profitant d'un contexte géographique idéal, l'économie de la logistique s'inscrit rapidement dans les représentations sociales comme le modèle à développer en région Nord-Pas-de-Calais et succède progressivement à l'économie industrielle minière. Ces paysages sont composés d'éléments issus de cette dynamique dominante de l'activité logistique, liée de fait à l'offre en infrastructures de transport du territoire.

En 1992 née l'idée d'un déplacement des activités de la gare Saint-Sauveur, située en plein centre de Lille, pour des raisons de nuisances et d'inadaptation. Les notables locaux imaginent sans difficulté, "naturellement», que le bassin minier puisse accueillir ces activités à forte nuisance. Comme l'indique un responsable du pôle d'excellence régional Euralogistic dont la mission, d'intérêt régional, se donne pour objectif d'« imposer le leadership logistique de la région Nord-Pas-de-Calais au nordouest de l'Europe »: "Le projet de développement de la filière logistique sur le territoire s'inscrit aussi dans une action en faveur des demandeurs d'emplois peu qualifiés. » Dans les discours que nous recueillons, la population du bassin minier est perçue comme ayant été de "tout temps" peu qualifiée. Il semble qu'elle doive le rester pour des édiles lillois qui ont choisi pour elle un type de développement économique, la logistique, activité dans laquelle la part de l'emploi peu qualifié est en hausse continue. Ce discours que nous avons entendu à de nombreuses reprises lors de nos investigations va de pair avec les indices de ce qui représente une expansion « naturelle » lilloise, et la prépondérance de la « ville-centre».

31 Ainsi, c'est également une histoire des rapports sociaux et politiques qui intervient ici. Comme le dit Alain Tarrius (1997), la perspective historico-anthropologique est toujours un élément majeur de compréhension des dynamiques d'aménagement, dans lesquelles se jouent et se rejouent de manière plus ou moins consciente les convergences et les antagonismes sociaux, les rapports de classes et les intérêts disparates constitués de longue date sur les territoires. Pour le dire platement et autrement, l'émergence d'une économie de la logistique et de ses paysages en ce lieu précis de la CAHC est due autant à la proximité de l'autoroute qu'à l'histoire des relations entre la ville de Lille et cette partie du bassin minier.

Figure 7. Parcelles agricoles sur la commune de Courcelles-lès-Lens

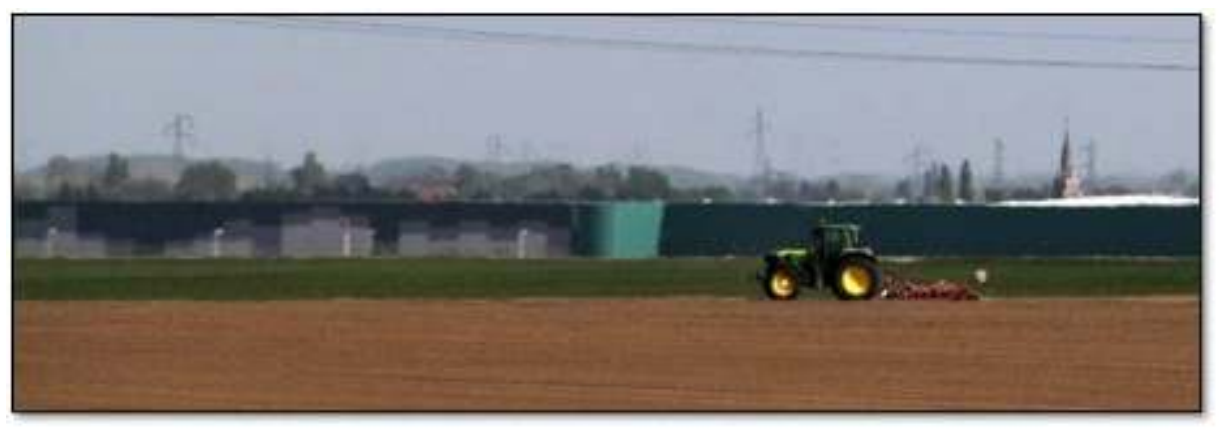

La logistique modifie les lignes d'horizon du territoire. Derrière les entrepôts de stockage Amazon apparaît ici le clocher de l'église de la commune de Lauwin-Planque, le beffroi de Douai, etc.

Source : Passeurs - Antoine Luginbühl. 
32 À l'échelle du territoire de la $\mathrm{CAHC}$, les répercussions paysagères de la plateforme multimodale sont profondes. Les aménagements logistiques génèrent des opportunités économiques et donc de nouvelles activités et pratiques de l'espace (le transbordement de marchandises au sein de la plateforme Delta 3 par exemple) et ils en amplifient d'autres, comme le trafic des poids lourds par exemple. Ces espaces de la logistique produisent donc une évolution des pratiques entre opportunités/facilités et contraintes/obligations. L'une des principales caractéristiques des aménagements liés à la logistique est la segmentation spatiale. Le paysage de Delta 3 est rationnel/ orthogonal/mathématique. La segmentation relève d'une forme géométrique générique, la ligne droite ou le rectilinéaire par distinction avec la courbe, le sinueux, voire le pli cher à Deleuze (1988). À l'intérieur des sites, nonobstant les ronds-points, le tracé des routes est droit, dessinant parfois des angles droits. Ce sont également des espaces plats, sans profondeur avec peu d'aspérités qui semblent déroulés comme le seraient des rouleaux de pelouses fabriqués pour les terrains de football. Il est notable par ailleurs, qu'au-delà de Delta 3 les espaces verts soient traités également de manière rectiligne : bandes de bitume, bandes de verdure, rangées d'arbres.

La segmentation est renforcée par la fermeture des espaces. Les dispositifs architecturaux de la segmentation ont ainsi pour rôle d'empêcher l'accès, de clore, de sécuriser les entrepôts contre le vol interne ou externe. Cette segmentation spatiale est rendue possible par des dispositifs architecturaux physiques ; le grillage, la barrière, les haies et les talus. L'inaccessibilité peut être renforcée par des dispositifs techniques : un poste de garde à l'entrée des entrepôts, des caméras de vidéosurveillance et une signalétique qui l'annonce. La sécurité et la protection sont au cœur de cette logique de la fermeture: protéger les marchandises, protéger les personnes d'espaces perçus comme dangereux (lieux de passage des trains, de transbordement de matériaux sensibles, voies ferrées, autoroutes). Priorité à la fluidité, celles des marchandises, celles des passagers qui vont vers Paris à $300 \mathrm{~km} / \mathrm{h}$, celles des usagers de l'autoroute. On n'arrête pas le TGV ni les camions pas plus que les péniches. La population locale passe en second. C'est elle qui doit enjamber les chemins clos de la mondialisation.

Figure 8. Entrepôts logistiques de la plateforme Delta 3

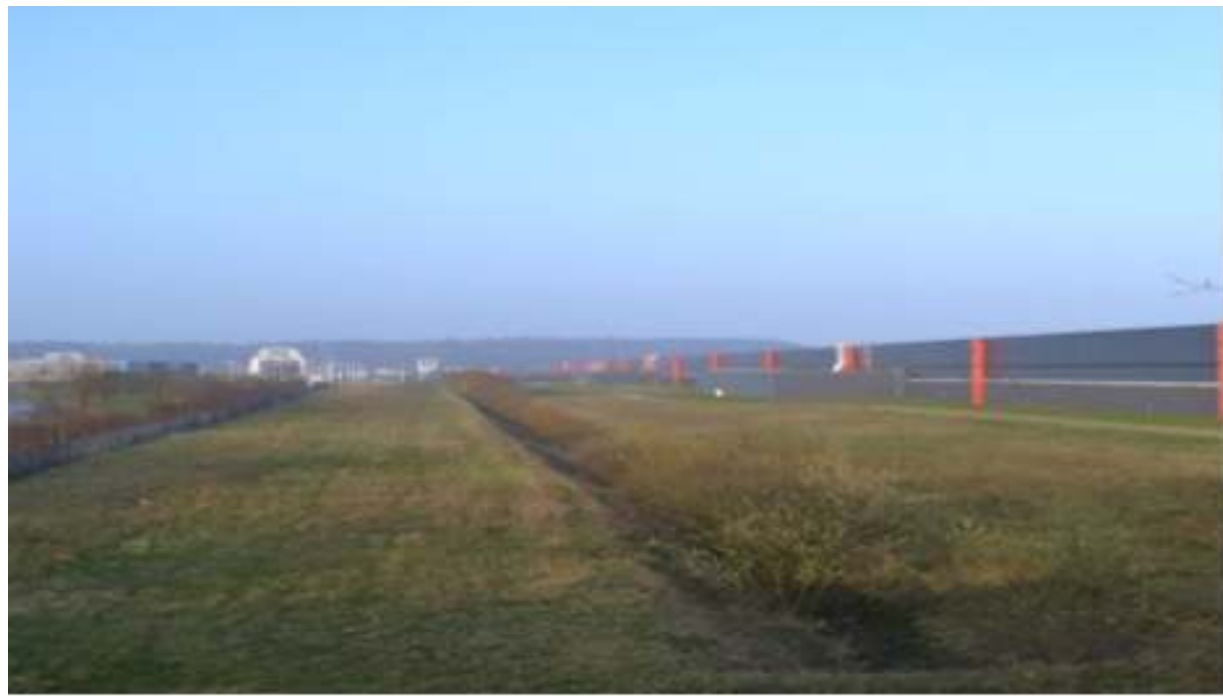

L'implantation d'infrastructures de stockage tend à structurer les paysages de la CAHC.

Source : Antoine Luginbühl. 
34

qui frappe le plus ainsi, c'est la rationalisation maximale de l'espace, comme si le territoire était devenu la carte. Lorsqu'un individu regarde une carte, il y projette surtout la necessité de se déplacer, une réponse à un besoin, selon une instrumentalisation de l'espace et non une interaction complexe. Et c'est bien de cela qu'il s'agit ici, se déplacer dans les espaces de la logistique, c'est se déplacer sur une carte grandeur nature, des traits blancs correspondant aux routes, des taches vertes aux forêts, du bleu pour l'eau. Comme s'il s'agissait de vivre sur le plan de l'aménageur. Sur la plateforme Delta 3, aucun entremêlement des fonctionnalités sociales, aucune habitation, aucun magasin, aucun terrain de sport, aucun lieu de culte, aucun bâtiment en fait, en dehors de ceux dédiés à la logistique.

\section{Conclusion}

e travail de caractérisation et d'analyse mené entre sociologues et paysagistes montre ainsi que les paysages de la $\mathrm{CAHC}$ se sont peu à peu construits à travers cinq grandes logiques de projet liées à l'agriculture, au patrimoine minier, à l'environnement, au phénomène de métropolisation et à la logistique. À chacune de ces différentes strates spatio-temporelles de constitution du territoire ne correspond pas un projet de paysage, mais une superposition de politiques sectorielles, interagissant plus ou moins les unes avec les autres. Les différentes politiques correspondent à des logiques d'acteurs par échelon territorial et aux niveaux de représentation de chaque échelle, tels que définis par Yves Luginbühl (2008). De ce point de vue, le paysage relève d'un processus interactif.

a conciliation du développement économique et du cadre de vie apparaît comme un enjeu majeur du territoire de la CAHC, lui permettant le renouvellement tant attendu de son image, s'appuyant sur la diversification de son modèle économique, la maîtrise et l'inscription de la dynamique logistique dans une politique globale d'aménagement du territoire.

Un des intérêts de l'association "sociologie et paysage " pour traiter les questions d'aménagement du territoire dans une démarche de participation citoyenne apparaît assez clairement au vu des résultats de notre recherche. Cette association permet de dépasser le projet d'aménagement ou l'étude sociologique pour proposer un projet politique transversal, issu d'une connaissance fine des interactions entre les formes du paysage, les jeux d'acteurs et les caractéristiques socioculturelles du territoire. Dans notre cas, il s'agit également de relier aménagement du territoire et modèle économique à partir de données quantifiables et non quantifiables, scientifiques et sensibles.

\section{BIBLIOGRAPHIE}

Ascher, F., Métapolis ou l'avenir des villes, Paris, Éditions Odile Jacob, 2010. 
Boltanski, L. et Thévenot, L., De la justification. Les économies de la grandeur, Paris, Éditions

Gallimard, coll. « NRF Essais », 1991.

Bourdin, A., Métapolis revisitée, La Tour d'Aigues, Éditions de l'Aube, 2014.

Cefaï, D., « Public, socialisation et politisation : Mead et Dewey », dans Cukier, A. et Debray, E. (dir.), La Théorie sociale de George Herbert Mead, Paris, La Découverte, 2013.

Dablanc, L., Frémont A., La Métropole logistique. Le transport de marchandises et le territoire des grandes villes, Paris, Édition Armand Collin, 2015.

Davis, M., City of quartz, Paris, La découverte poche, coll. « Sciences humaines et sociales », 2006.

Delaporte, Y., « D’un terrain l'autre. Réflexions sur l'observation participante », dans Pétonnet, C. et Delaporte, Y. (textes réunis par), Ferveurs contemporaines. Textes d'anthropologie urbaine offerts à Jacques Gutwirth, Paris, L'Harmattan, 1993, p. 321-340.

Dubois-Tainé, G., Chalas, Y. (dir.), La Ville émergente, La Tour d'Aigues, Éditions de l'Aube, 1997, $286 \mathrm{p}$.

Hannerz, U., Explorer la ville. Éléments d'anthropologie urbaine, Paris, Les Éditions de Minuit, coll. « Le sens commun », 1983.

Joseph, I., La Ville sans qualités, La Tour d'Aigues, Éditions de l'Aube, 1998.

Luginbühl, Y., « Las representaciones del paisaje y sus evoluciones », dans Maderuelo, J. (dir.), Paisaje y territorio, Huesca, CDAN Editores, 2008, p. 143-180.

Mattei, M.-F., « Appel à proposition de recherche : la ville ordinaire et la métropolisation », DGALN, Plan urbanisme construction architecture, ministère de l'Écologie, du Développement durable et de l'Énergie, 2013.

Peraldi, M., « Rapport d'échange et ordre moral : l'épaisseur sociale de la grande surface : le cas de Plan de Campagne », Lames-MMSH-CNRS, 2001.

Petonnet, C., On est tous dans le brouillard, Paris, éditions Galilée, 1979.

Petonnet, C., Espaces habités, Paris, éditions Galilée, 1982.

Sassen, S., The Global City: New York, London, Tokyo, Princeton, Princeton University Press, 1991.

Savy, M., " La logistique en France : État des lieux et pistes de progrès », rapport du comité scientifique de la Conférence nationale sur la logistique présidé par Michel Savy, 2015.

Tarrius, A., Fin de siècle incertaine à Perpignan, Perpignan, Éditions Trabucaire, 1997.

Tarrius, A., Les Fourmis d'Europe : migrants pauvres et nouvelles villes internationales, Paris, L'Harmattan, 1992.

Tarrius, A., Expérience pour une anthropologie du mouvement : d'une sociologie des transports à une anthropologie de la mobilité spatiale, Caen, Paradigme, 1989.

\section{NOTES}

1. La communauté d'agglomération d'Henin-Carvin (CAHC) est une intercommunalité regroupant 14 communes, située dans le département du Pas-de-Calais et de la Région Nord-Pas-de-Calais.

2. Au sens de Luc Boltanski et Laurent Thévenot 1991), le projet « rassemble temporairement des personnes très disparates, et se présente comme un bout de réseau fortement activé pendant une 
période relativement courte, mais qui permet de forger des liens plus durables qui seront ensuite mis en sommeil tout en restant disponibles » (1991, p. 157).

3. Voir Notamment Diane Burgess, Graham Finney, Dave Matthews, Miles Patton, in 1st International Conference on Landscape Economics, Vienna Austria, 2009.

4. Ittecop est un programme de recherche du ministère de l'Écologie, du Développement durable et de l'Énergie (Medde), en coordination avec l'Agence de l'environnement et de la maîtrise de l'énergie (Ademe). L'objectif principal visait à confronter les enjeux techniques des ILTE (Infrastructures de transport et leurs emprises) routières, ferrées, fluviales ou énergétiques, et leurs interfaces avec les territoires (gares, ports, aéroports, etc.) en incluant les dimensions paysagères et écosystémiques.

5. Des approches où les typologies paysagistes voisineraient avec les critiques de toute tentative de définition objectivante d'un paysage.

6. La démarche méthodologique mobilisée par l'équipe de socio-anthropologues se situe dans la tradition de l'anthropologie sociale et culturelle qualitative anglo-saxonne. L'objectif est de s'approcher au plus prés des «situations naturelles des sujets" afin de produire des connaissances in situ, contextualisées, permettant de rendre compte du "point de vue de l'acteur", des représentations ordinaires, des pratiques usuelles et de leurs significations autochtones.

7. Nous nous situons ici dans la continuité des approches de socio-anthropologie urbaine anglosaxonnes, représentées en France par les travaux d'Alain Tarrius, de Daniel Céfaï ou Michel Péraldi.

8. «En effet, les constructions neuves se réalisent pour près des deux tiers hors d'opération d'urbanisme; les maisons individuelles représentant $60 \%$ de l'ensemble » dans Marie-Flore Mattei (2013).

9. Selon Daniel Céfaï (20123): «L'activité collective qui se forme autour d'une situation problématique a été analysée, dès lors qu'elle engage des personnes qui ne sont pas directement concernées, en termes de constitution d'un public autour d'un problème public [...]. Une situation est réfléchie comme problématique lorsqu'elle organise l'activité collective de ses participants, alors qu'ils s'efforcent d'en déterminer, en vue de les maîtriser, les conditions de son advenue, les phases de son déroulement et les conséquences qui s'ensuivent. »

10. Les travaux d'Alain Tarrius montrent comment les mobilités et leurs réseaux «travaillent l'espace ", s'y inscrivent, le qualifient, le constituant en ressources et en territoire. Voir notamment, Alain Tarrius (1992 et 1989).

11. Le terme " cavalier " désigne les voies de chemin de fer reliant les exploitations aux mines et les exploitations entre elles, permettant l'acheminement des extractions par des wagonnets.

12. Ce qui s'illustre pleinement dans le discours des promoteurs touristiques de la CAHC: «Ce qu'il y a de bien dans le bassin minier, c'est le paradoxe! Du noir, on passe au vert, du souterrain de la mine, si profonde, on passe au ciel, en grimpant en haut d'un chevalet ou d'un terril ! Tel un aventurier à l'assaut d'une mine d'or, j'aime partir explorer ce vrai terrain d'aventure de tous les temps : d'abord l'aventure humaine, puis industrielle et maintenant sportive pour le randonneur que je suis! » (Source : http://www.tourisme-nord.fr/) 


\section{RÉSUMÉS}

À partir d'une recherche menée depuis trois ans dans le cadre du programme Ittecop (ministère de l'Écologie-Ademe) ce texte propose une analyse des paysages de la communauté d'agglomération d'Hénin-Carvin (CAHC) située dans le bassin minier du Nord-Pas-de-Calais. Après avoir présenté les enjeux méthodologiques d'une analyse de la dimension paysagère des projets d'aménagement du territoire, le texte détaille cinq dynamiques paysagères de cette partie du bassin minier: entre passé minier, enjeux environnementaux et développement récent de l'activité économique de logistique des marchandises. Au centre du propos se trouve l'idée que le processus continu de production des paysages relève moins de la mise en œuvre d'un projet que de la succession et de l'entremêlement de projets de territoire portés par différents acteurs en interaction. Des acteurs qui ne sont pas toujours politiques, mais qui sont principalement focalisés sur des enjeux de développement économique. Des acteurs qui, surtout ici, traitent de manière secondaire les enjeux paysagers, inscrits dans différentes profondeurs historiques et articulant plusieurs échelles.

Based on research conducted during three years as part of the Ittecop programme (French Ministry of Ecology and French Agency for the Environment and Energy - ADEME) this paper presents a study of the landscapes of the Hénin-Carvin grouping of municipalities (in French, Communauté d'agglomération d'Hénin-Carvin - CAHC) located in the Nord-Pas-de-Calais mining area. After presenting the methodological issues involved in an analysis of the landscape dimension in regional planning projects, the article describes the landscape dynamics of projects in this part of the mining region which combine the mining heritage, environmental issues and the recent development of economic activity in the freight transport sector. At the heart of the debate is the notion that the continuous process of the production of landscapes has less to do with the implementation of a project than with the successive inter-meshing of regional development projects supported by different interacting stakeholders. These stakeholders may not always be politicians but they are, however, mainly focused on economic development issues. In this particular case, the stakeholders consider landscape issues relating to different historical levels and providing links on different scales to be of secondary importance.

\section{INDEX}

Mots-clés : paysages stratifiés, acteurs et aménagement du territoire, bassin minier, Nord-Pasde-Calais, dispositif paysager

Keywords : composite landscapes, regional development stakeholders, mining area, Nord-Pasde-Calais, landscape system

\section{AUTEURS}

\section{FABRICE RAFFIN}

Fabrice Raffin est maître de conférence en sociologie à l'université de Picardie Jules Verne, Amiens. fbraffin[at]gmail[dot]com 


\section{ANTOINE LUGINBÜHL}

Antoine Luginbühl est paysagiste DPLG et cogérant de la structure Passeurs, Paysage et participation citoyenne (Bordeaux, Chambéry).

antoine.luginbuhl[at]free[dot]fr

www.passeurs.eu

\section{OLIVIER POLLET}

Olivier Pollet est sociologue, il est chargé de recherches à SEA Europe, Paris.

polletolive[at]gmail[dot]com 